\title{
WiFi-Based Virtual Access Network Scheduling for Downlink Traffic Dominated Smart Spaces
}

\author{
Pin Lv $\mathbb{C D}^{1,2,3}$ Siyu Pan $\mathbb{D}^{1}{ }^{1}$ and Jia Xu $\mathbb{D}^{1,2,3}$ \\ ${ }^{1}$ School of Computer Electronics and Information, Guangxi University, Nanning 530004, China \\ ${ }^{2}$ Guangxi Key Laboratory of Multimedia Communications and Network Technology, Nanning 530004, China \\ ${ }^{3}$ Guangxi Colleges and Universities Key Laboratory of Parallel and Distributed Computing, Nanning 530004, China
}

Correspondence should be addressed to Jia Xu; xujia@gxu.edu.cn

Received 25 March 2020; Revised 27 July 2020; Accepted 24 October 2020; Published 6 November 2020

Academic Editor: Sungchang Lee

Copyright (@ 2020 Pin Lv et al. This is an open access article distributed under the Creative Commons Attribution License, which permits unrestricted use, distribution, and reproduction in any medium, provided the original work is properly cited.

\begin{abstract}
WiFi networks are widely and densely deployed as infrastructure in smart spaces. However, differentiated services with guaranteed access bandwidths are not supported in traditional WiFi networks. In this paper, wireless virtual access networks are established to provide guaranteed downlink bandwidths for primary users. For each primary user with a demanded access bandwidth, a group of APs are coordinated to serve it. In order to maximize network utilization, two wireless virtual access network scheduling algorithms are designed. One scheduling algorithm is designed based on the maximum independent set in the conflict graph, which has an exponential computation complexity. The other scheduling solution is based on a greedy strategy with linear complexity. Simulation results prove that both scheduling algorithms improve network utilization effectively, and the greedy algorithm is more suitable for practical use.
\end{abstract}

\section{Introduction}

As important infrastructure, WiFi networks are widely and densely deployed in many smart space scenarios $[1,2]$, such as smart home [3], smart building [4], and smart campus [5]. WiFi-enabled smart devices access the Internet as stations when they are associated with WiFi access points (APs). Since WiFi networks are based on IEEE 802.11 standards, distributed coordinate function (DCF) is adopted as the media access control (MAC) mechanism in most cases. With such MAC mechanism, all wireless nodes including both APs and stations have almost equal opportunity to randomly access the channel, and the quality of service (QoS) cannot be guaranteed due to contention and backoff. In many smart space cases, downlink traffic is far more than uplink traffic, and some users demand various but guaranteed downlink bandwidths. For example, a 4-channel HD (high-definition) monitor may demand a guaranteed downlink bandwidth of $16 \mathrm{Mbps}$, and a remotely controlled device may only require a downlink bandwidth of $100 \mathrm{kbps}$ which is also guaranteed. Traditional WiFi networks cannot support such differentiated and guaranteed service. Hence, wireless network virtualization is introduced into $\mathrm{WiFi}$ networks to meet the personalized bandwidth requirements of the users.

Wireless network virtualization is an emerging technology which has attracted much attention in recent years $[6,7]$, and WiFi network virtualization is studied for service isolation [8] or seamless roaming [9]. With wireless network virtualization, network infrastructure can be decoupled from the services that it provides, where differentiated services can coexist on the same infrastructure, maximizing its utilization [10]. In order to provide guaranteed QoS for the users, virtual networks are established and operated in isolation from each other. In order to manage and maintain virtual networks automatically, software-defined networking (SDN) technology is utilized to control network resources for data transmission [11]. At least one controller is deployed in SDN to take charge of resource allocation.

In this paper, two types of WiFi network users in downlink traffic dominated smart space are considered, which are the primary users and the secondary users. The primary users have specific access bandwidth requirements, 
and the secondary users do not have such requirements. Wireless virtual access networks (VANs) are established in software-defined WiFi networks to provide primary users with differentiated and guaranteed access bandwidths. The residual bandwidth is allocated to the secondary users to provide them the best-effort service. In order to improve network utilization, the VANs should be scheduled subtly to maximize the residual bandwidth. Hence, two scheduling algorithms are designed. In the first scheduling algorithm which is called MISS, most VANs without conflict are found out based on the maximum independence set to transmit concurrently, which has an exponential computation complexity. The second scheduling algorithm is a greedy algorithm which has a linear computation complexity; thus, it is named LINS. Extensive simulations are conducted to evaluate the performances of MISS and LINS. Based on the simulation result analysis, MISS outperforms LINS slightly in network utilization maximization, but LINS has higher efficiency than MISS.

The contributions of this paper are summarized as follows:

(1) A framework of the wireless virtual access network is proposed for downlink traffic dominated WiFi networks to provide differentiated services with guaranteed downlink bandwidths

(2) Two wireless virtual access network scheduling algorithms, i.e., MISS and LINS, are designed to maximize the WiFi network utilization

(3) A simulator is developed to evaluate the performances of the two scheduling algorithms, and a conclusion is drawn that LINS is more suitable for practical use

The remainder of the paper is organized as follows. Related work is summarized in Section 2. The system model is described in Section 3. In Section 4, two VAN scheduling algorithms are designed. Experimental results are analyzed in Section 5, and the paper is concluded in Section 6.

\section{Related Work}

Wireless network virtualization is considered a promising technology to improve resource utilization, QoS, security, and so forth, which has become a research hotspot in recent years $[6,12,13]$. In wireless network virtualization, a logical function is decoupled from the physical resource. According to the mapping relation between the physical resource and logical function, the related work can be divided into three types, that is, isolation, aggregation, and hybrid.

In the isolation type of wireless network virtualization, multiple virtual networks coexist on the same physical wireless network without mutual interference. It is a one-tomany relationship from a physical network to virtual networks. The virtual networks are isolated from each other in at least one dimension, such as time, frequency, space, and coding [14]. In [8], the virtual access network in the wireless mesh network is proposed to provide guaranteed access bandwidth, and the virtual access networks are assigned different OFDMA subcarriers for isolation. In this paper, virtual access networks are constructed in commodity WiFi networks to provide primary users with guaranteed access bandwidths. The distinct feature of this paper is that virtual access networks are isolated in the time domain, and two scheduling algorithms are designed correspondingly.

In the aggregation type of wireless network virtualization, multiple physical devices serve as a virtual entity together. It is a many-to-one relationship from physical devices to virtual entity. For example, in [15], a mechanism that multiple APs are virtualized into a virtual AP is described. With the help of virtual AP, seamless roaming within the WiFi network is achieved, and AP diversity is employed to enhance the transmission rate. In this paper, we leverage the similar AP virtualization mechanism with [15] but extend it to support multiple virtual access networks with guaranteed bandwidths.

The hybrid type of wireless network virtualization is a combination of the above two types, in which the physical networks and virtual networks have a many-to-many relationship. In [16], for instance, the isolated virtual networks are supported by heterogeneous physical networks. The wireless network virtualization scheme in this paper also belongs to the hybrid type. In our proposed scheme, multiple APs are virtualized into a single AP, while the physical WiFi network accommodates multiple virtual access networks.

To the best of our knowledge, this is the first attempt to combine the virtual access network with AP aggregation virtualization.

\section{System Model}

In this paper, downlink traffic dominated smart space scenario is considered. In the scenario, a software-defined WiFi network is deployed to provide Internet access to devices. In the software-defined WiFi network, all the APs are managed by a controller. The wireless interfaces of all the APs are configured into identical parameters, including IP address, MAC address, channel, and ESSID. Hence, multiple APs act as a unique virtual AP [15]. If the APs are set to different nonoverlapping channels to increase the network capacity, the APs on the same channel are organized into a virtual AP. In this case, multiple virtual APs exist in the WiFi network, and each virtual AP operates in the way described in this paper. When a station is associated with the virtual $\mathrm{AP}$, it accesses the Internet through the WiFi network. If the station transmits a packet to the virtual AP, all the APs within its transmission range receive it. These APs are organized into a group to serve the station. The AP group forms a wireless virtual access network (VAN) for the station. Each AP in the group periodically reports the signal strength of the station to the controller. When a downlink packet is needed to send to the station, it is firstly multicast to each member in the AP group, and one AP from the group is chosen by the controller to transmit the packet to the station according to the signal strength. Meanwhile, the controller commands other APs which may cause collisions to keep silent. Without the controller, multiple APs may contend for the channel to transmit packets, and collisions may cause 
serious performance degradation. In order to avoid a collision caused by uplink transmissions, the APs always set the contention window to 0 . Therefore, the downlink transmission has a higher priority than the uplink transmission.

The users in the WiFi network are classified into two types, i.e., the primary users (PUs) and the secondary users (SUs). The primary user $\mathrm{PU}_{i}$ has an access bandwidth requirement $b_{i}$, which should be guaranteed. In this paper, the normalized bandwidth is used to facilitate the analysis. For example, if a primary user demands an access bandwidth of 0.2 , it means $20 \%$ of the access time should be allocated to the user. It is assumed that the sum of all primary users' demanded normalized bandwidth does not exceed 1 . Hence, for each primary user, its VAN is assigned a certain active time $A_{i}$. In the active time, the AP group transmits packets to the primary user, and the VANs of other stations should not interfere with the transmission. It is feasible to guarantee access bandwidth by means of active time allocation $[17,18]$. The advantage of our solution is that it is compatible with IEEE 802.11, and no modification is needed on the user-side devices.

An example is demonstrated in Figure 1. Four APs and two primary users exist in a WiFi network. The VAN of PU1 contains AP1 and AP2, and the AP group which serves PU2 includes AP2, AP3, and AP4. Since AP2 exists in the two AP groups at the same time, the VANs of PU1 and PU2 cannot be activated simultaneously.

The active time allocated to a primary user may contain several separated time intervals as follows:

$$
A_{i}=a_{i 1} \cup a_{i 2} \cup, \ldots, \cup a_{i n} .
$$

The total length of these time intervals should be equal to the access bandwidth requirement of the primary user, i.e.,

$$
b_{i}=\sum_{j=1}^{n} L\left(a_{i j}\right) .
$$

After catering to the bandwidth requirements of all the primary users, the residual bandwidth is used by the secondary users, as shown in Figure 1. The residual bandwidth is denoted as $B_{\mathrm{s}}$ (bandwidth for secondary users), and the total bandwidth for primary users is $B_{\mathrm{p}}$. They are computed as follows:

$$
\begin{aligned}
& B_{\mathrm{s}}=1-B_{\mathrm{p}}, \\
& B_{\mathrm{p}}=S\left(\left\{b_{i} \mid i=1,2, \ldots, m\right\}\right),
\end{aligned}
$$

where $S\left(\left\{b_{i} \mid i=1,2, \ldots, m\right\}\right)$ means the total bandwidth allocated to $m$ primary users under a certain scheduling scheme.

In order to improve the network utilization, the VANs should be scheduled skillfully to maximize the residual bandwidth. Therefore, the optimization objective is to maximize the residual bandwidth as follows:

$$
\max B_{s} \text {. }
$$

From another perspective, the concurrent transmissions of different VANs should be maximized, and the active time for all primary users is minimized using appropriate scheduling:

$$
\min B_{\mathrm{p}} .
$$

To determine whether VANs can transmit concurrently, a conflict graph should be constructed and maintained by the controller. In the conflict graph, a node represents a VAN. If two VANs conflict, an edge exists between the corresponding nodes.

Denote the interference radius of the AP as $R_{i}$. Two VANs do not interfere with each other if their distance is larger than $R_{i}$. The distance between two VANs, $V_{1}$ and $V_{2}$, is defined as

$$
D\left(V_{1}, V_{2}\right)=\min _{n_{i} \in V_{1}, n_{j} \in V_{2}} d\left(n_{i}, n_{j}\right),
$$

where $d\left(n_{i}, n_{j}\right)$ is the distance between wireless nodes $n_{i}$ and $n_{j}$ and a wireless node in a VAN is either an AP or a station.

\section{Wireless Virtual Access Network Scheduling Algorithms}

Finding optimal scheduling for such an environment is an NP-hard problem [19]; two VAN scheduling algorithms are designed to find approximate optimal solutions. The first scheduling algorithm is based on the maximum independent set, and it is referred to as MISS. The second one is a greedy algorithm which has linear complexity, thus it is called LINS. The scheduling algorithm is executed by the controller.

4.1. MISS. The MISS algorithm explores the confliction relationships between the VANs of the primary users based on the conflict graph of the network. The maximum independent set of the conflict graph indicates the largest set of VANs in which any VAN does not interfere with each other. Thus, these VANs can be activated at the same time.

As shown in Algorithm 1, in the scheduling process, the maximum independent set of the conflict graph is found. Each VAN in the maximum independent set is allocated a time interval with the length equal to the shortest bandwidth requirement in the set. If the bandwidth requirement of $v_{i}$ (the VAN for $\mathrm{PU}_{i}$ ) is satisfied, it is removed from the conflict graph. Repeat the process until all the VANs are provided their demanded bandwidths.

From Lines 1 to 4, the allocated active time and bandwidth requirement of each VAN are initialized. Line 5 indicates the termination condition of the loop is that each VAN is assigned enough service time. In Line 6, the maximum independent set is computed, which means the most VANs without mutual interference are found. Among these VANs, the one with the least unsatisfied bandwidth requirement determines the active interval length of the VANs in the maximum independent set. Hence, the length of the least unsatisfied bandwidth requirement is obtained in Line 7. From Lines 8 to 15 , every VAN in the maximum independent set is allocated an active interval. If the bandwidth requirement of a VAN is satisfied, it is removed from the candidate set. 


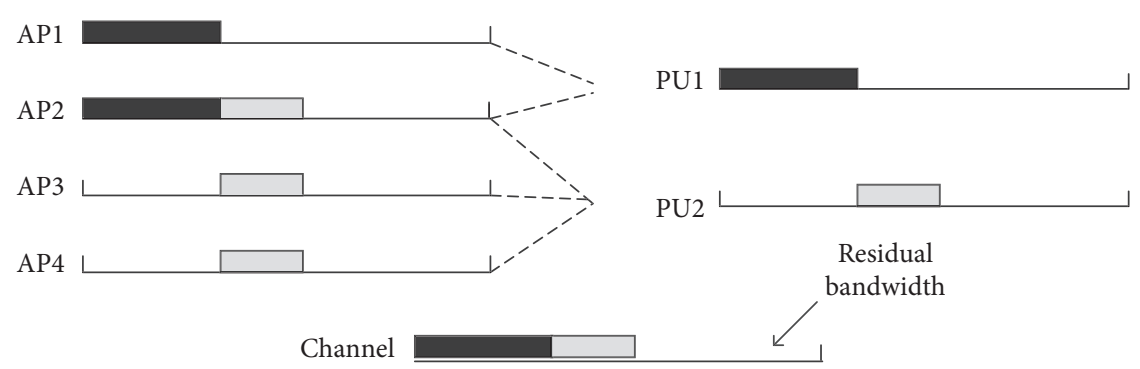

(a)

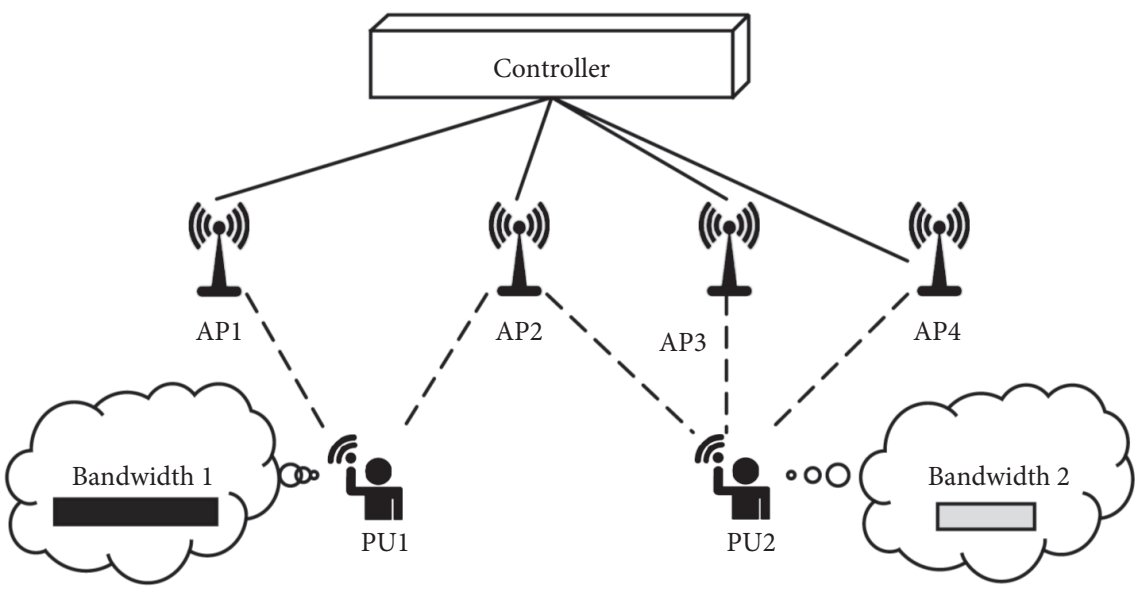

(b)

Figure 1: An example of wireless virtual access networks.

Input: conflict graph $G=\langle V, E\rangle$, bandwidth requirement $b$ of each PU in ascending order

Output: active time $A_{i}$ for each $v_{i} \in V$

(1) beginTime $=0$

(2) for each $v_{i} \in V$ do

(3) $A_{i}=\phi$;

(4) $\quad v_{i}$ length $=b_{i}$;

(5) while $V \neq \phi$ do

(6) Find out the maximum independent set $M$ in $G$;

(7) Find out $v_{\min } \in M$ with the least length;

(8) for each $v_{i} \in M$ do

(9) $\quad a_{j}$ length $=v_{\min }$ length;

(10) $\quad a_{j}$ begin = beginTime;

(11) beginTime $=$ beginTime $+a_{j}$ length;

(12) $\quad A_{i}=A_{i} \cup a_{j}$;

(13) $\quad v_{i}$ length $=v_{i}$ length $-v_{\min }$ length;

(14) if $v_{i}$ length $=0$ then

(15) $\quad V=V-\left\{v_{i}\right\}$;

Algorithm 1: MISS.

Since the process of finding the maximum independent set has a complexity of $O\left(2^{n}\right)$, the computation complexity of MISS is $O\left(2^{n}\right)$, which is exponential.

4.2. LINS. To achieve low computation complexity, LINS is designed based on a greedy strategy. LINS schedules the VANs in the descending order of demanded bandwidths.
The VAN with the largest demanded bandwidth is allocated active time first. For the following VAN, it is assigned as early as possible active time in which it does not interfere with other scheduled VANs, as shown in Algorithm 2. Different from MISS, each VAN is allocated a continuous service time interval under the scheduling of LINS.

Lines 1 to 4 are also an initialization process. Since the VANs are scheduled in the descending order of the 


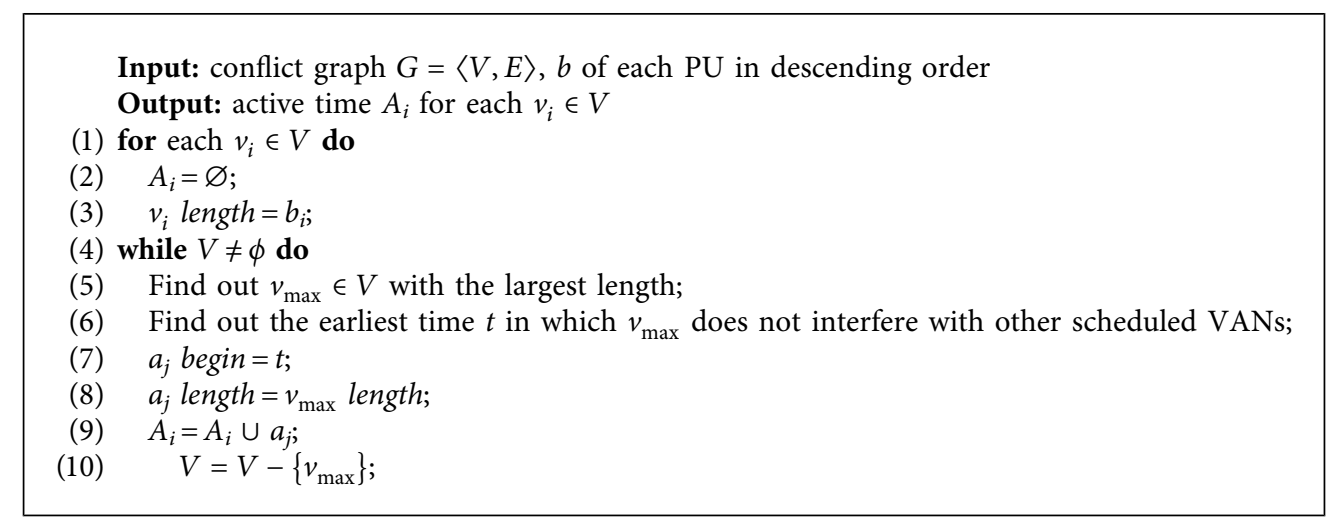

Algorithm 2: LINS.

bandwidth requirement, the VAN with the largest demanded bandwidth is fetched in Line 5, and it is allocated a service time as early as possible from Lines 6 to 10 .

The computation complexity of LINS is $O(n)$, which is linear.

4.3. Case Study. Suppose four VANs coexist in a WiFi network, and the conflict graph of them is demonstrated in Figure 2. Each node in the conflict graph represents a VAN. If a line connects two nodes, it means the corresponding VANs interfere with each other.

The demanded bandwidths of the four primary users are $0.3,0.3,0.2$, and 0.2 , respectively. The scheduling schemes under MISS and LINS are shown in Figure 3.

For MISS, a maximum independent set containing User 1 and User 3 is found firstly, according to the interference relation. In this maximum independent set, User 3 has the least demanded bandwidth. Hence, the two users are allocated an active interval with the length of 0.2 , which is the demanded bandwidth of User 3. After this interval, the bandwidth requirement of User 3 has been satisfied, and it is removed from the conflict graph. In the next round of the loop, another maximum independent set containing User 1 and User 4 is found. Since the remainder unsatisfied bandwidth requirement of User 1 is 0.1 , which is lower than the demanded bandwidth of User 4, the two users are allocated an active interval with the length of 0.1 . After that, User 1 is removed from the conflict graph because the total length of the allocated active intervals equals its demanded bandwidth. In the next round, a maximum independent set containing only User 2 is found, and it is allocated an interval with the length of 0.3 . At last, User 4 is the only node left in the conflict graph, and it is allocated an interval to satisfy its remainder demanded bandwidth. A feature of MISS is that the demanded bandwidth of a user may be divided into multiple separated intervals, like User 4 in this case.

For LINS, the primary users are allocated active intervals in the descending order of the demanded bandwidth, and the interval is not divided into separated parts. In this case, User 1 is allocated an interval with the length of 0.3 first. Next, User 2 is allocated the following interval with the

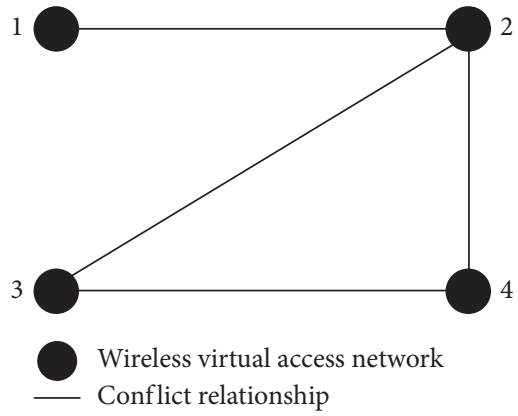

Figure 2: An example of the conflict graph.

length of 0.3 , due to the fact that it has a conflict relation with User 1 . When User 3 is considered, since it does not interfere with User 1, it is allocated a concurrent interval with User 1. At last, User 4 is allocated an interval after User 2's interval, because an earlier continuous interval cannot be found.

Besides the bandwidth allocated to primary users, the residual bandwidth can be used by secondary users. In this case, the residual bandwidth of MISS is 0.3 , while the residual bandwidth of LINS is 0.2 . Hence, more bandwidth is residual under the scheduling of MISS, and the WiFi network has a higher utilization.

It is revealed from the case that MISS has a more finegrained scheduling than LINS, but LINS has higher efficiency.

\section{Performance Evaluation}

A simulator is developed, and extensive simulations are carried out to evaluate the performance of the proposed VAN scheduling algorithms.

5.1. Experiment Setup. A WiFi network with 25 APs and 50 primary users is generated in the simulator. The APs are deployed as a $5 \times 5$ grid in a $500 \mathrm{~m} \times 500 \mathrm{~m}$ rectangular area. The primary users distribute randomly in the area, following a uniform distribution. Both the transmission range and the interference range of the wireless node follow a disk model, and the transmission radius is $100 \mathrm{~m}$ and the interference 


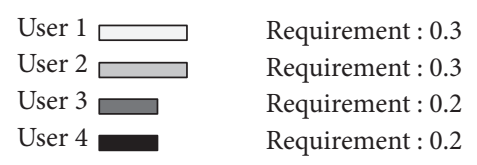

(a)

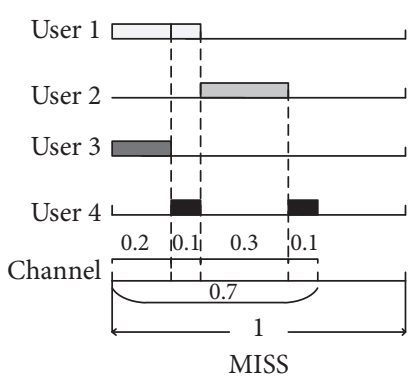

(b)

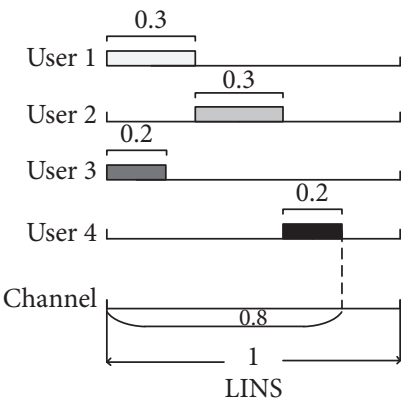

(c)

FIgURE 3: Scheduling comparison of MISS and LINS.

radius is $150 \mathrm{~m}$. The default parameters in the simulations are listed in Table 1.

A benchmark is introduced as a comparison with MISS and LINS, which is called SUM. The SUM is the sum of the required bandwidths of all primary users without considering the possible overlaps between them as follows:

$$
\mathrm{SUM}=\sum b_{i}
$$

To evaluate the performance from multiple perspectives, the following metrics are measured:

(1) For the sake of fair comparison, optimized service time for all primary users to that of SUM ratio (OTS) is presented as a new metric (computed as the following equation):

$$
\mathrm{OTS}=\frac{\mathrm{O}}{\mathrm{SUM}}
$$

where $O$ is the total service time for primary users under the scheduling of MISS or LINS. OTS is a ratio between 0 and 1 , and it should be minimized.

(2) The average time costs for scheduling (i.e., the time for program execution) should be minimized.

(3) The residual bandwidth for secondary users should be maximized.

5.2. Experiment Results. When the number of primary users varies from 10 to 100, the OTSs of MISS and LINS are shown in Figure 4. The OTSs of both MISS and LINS range from $40 \%$ to $50 \%$ and do not change obviously with the increase in the number of primary users. In each case, the OTS of MISS is slightly lower than that of LINS ( $2 \%$ on average), which indicates that MISS outperform LINS only a little.

When changing the distances between the adjacent APs from 50 meters to 150 meters, the OTSs of MISS and LINS are shown in Figure 5. As the AP density decreases, the OTSs of the two scheduling algorithms decline slowly. This is because when the distance between APs increases, the transmission conflicts among AP groups reduce, and more concurrent transmissions are available. The OTSs of MISS are smaller than those of LINS, and the differences between MISS and LINS are still limited.
Table 1: Default parameters in the simulations.

\begin{tabular}{lc}
\hline Parameter & Default value \\
\hline Number of APs & 25 \\
Number of primary users & 50 \\
Demanded bandwidth of primary users & 0.02 \\
Distance between adjacent APs (m) & 100 \\
Transmission radius of AP (m) & 100 \\
Interference radius of AP (m) & 150
\end{tabular}

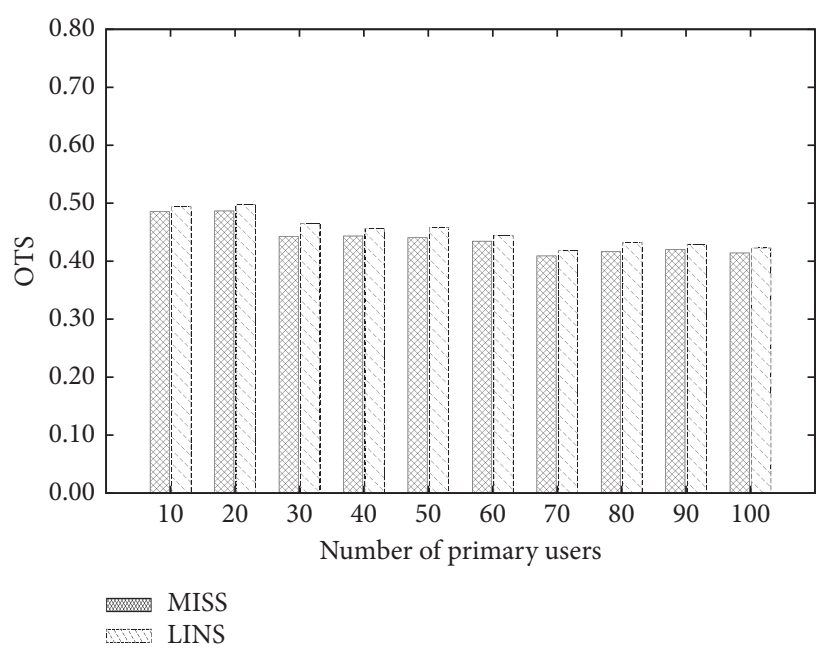

FIgURE 4: OTS of MISS and LINS when the number of primary users varies.

In Figure 6, the OTSs of MISS and LINS are displayed when the demanded access bandwidth scope of primary users changes. From the results, it can be seen that the demanded access bandwidth scope of primary users does not affect OTSs of the two scheduling algorithms.

From Figures 4-6, it is concluded that MISS has lower OTS than that of LINS when different parameters change. However, the differences between the two scheduling algorithms are quite small.

The average time costs for scheduling under different circumstances are also measured. In Figure 7, when the number of primary users increases from 10 to 100 , the time cost of MISS surges exponentially, while the time cost of 


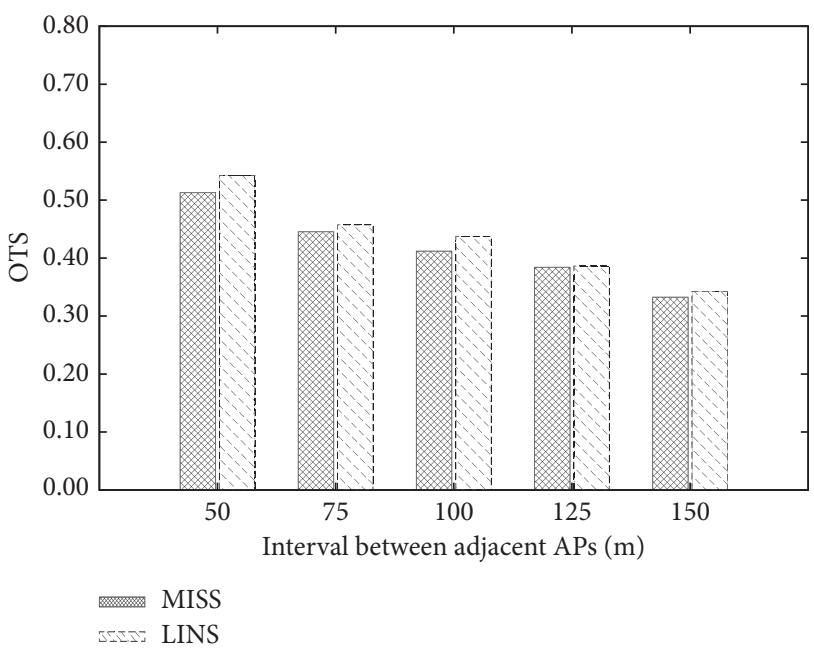

Figure 5: OTS of MISS and LINS when the distance between the adjacent APs varies.

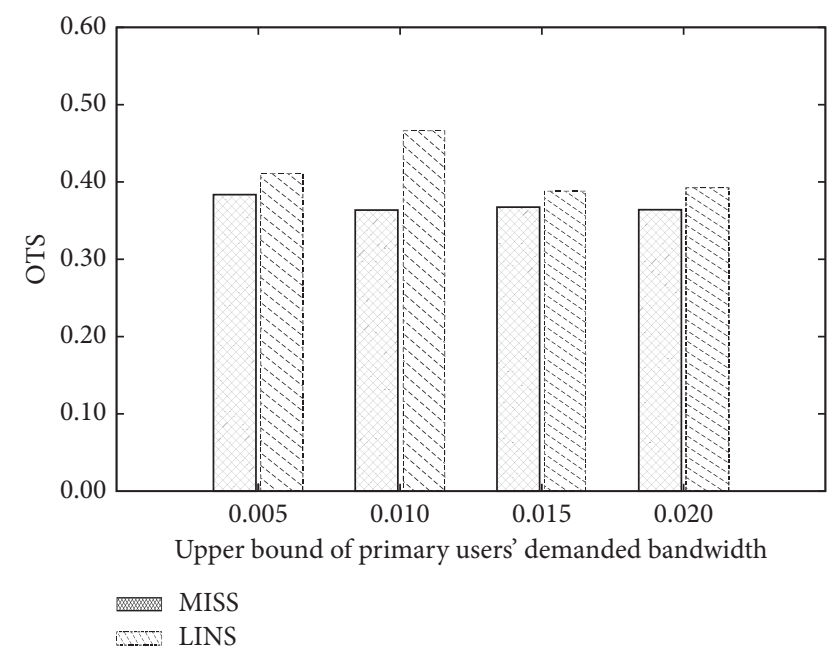

Figure 6: OTS of MISS and LINS when the demanded bandwidth of primary users varies.

LINS has a slight linear increment. The different computation complexities of the two scheduling algorithms lead to this result.

The time costs relative to AP densities are shown in Figure 8 . The time cost of MISS still has obvious growth. In this case, however, the time cost of LINS even decreases slowly, because transmission conflicts reduce.

When the demanded bandwidth scope of the primary users becomes larger, the time cost of MISS has a slight increment, while the time cost of LINS keeps stable, as depicted in Figure 9. The differences between the two algorithms are still large.

From Figures 7-9, it is concluded that the time cost of MISS grows much faster than that of LINS. The difference is obvious especially when the scale of the WiFi network is large. The reason is that MISS has an exponential computing complexity, and LINS has a linear complexity. Since the two

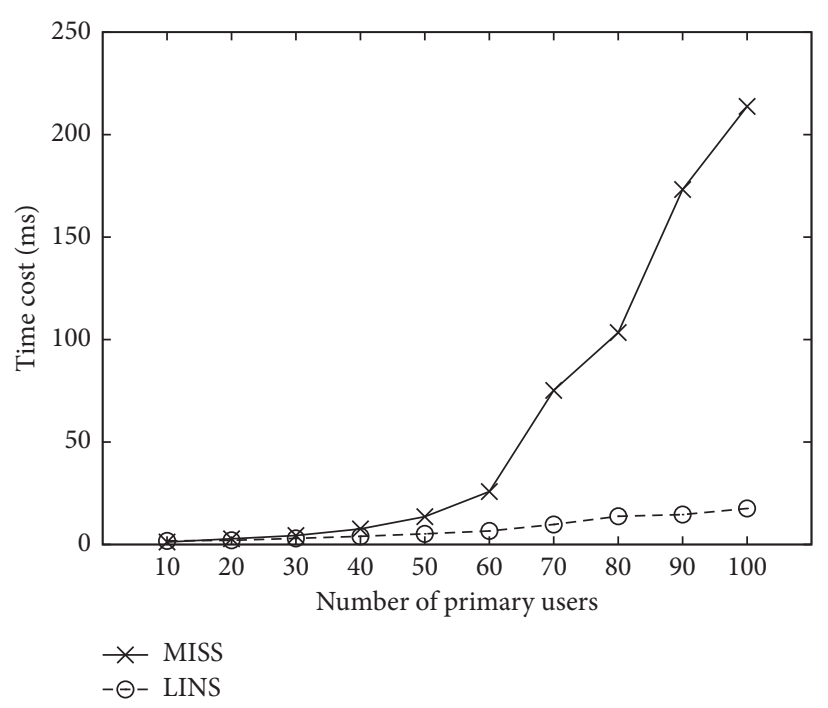

FIgURE 7: The average time cost of MISS and LINS when the number of primary users varies.

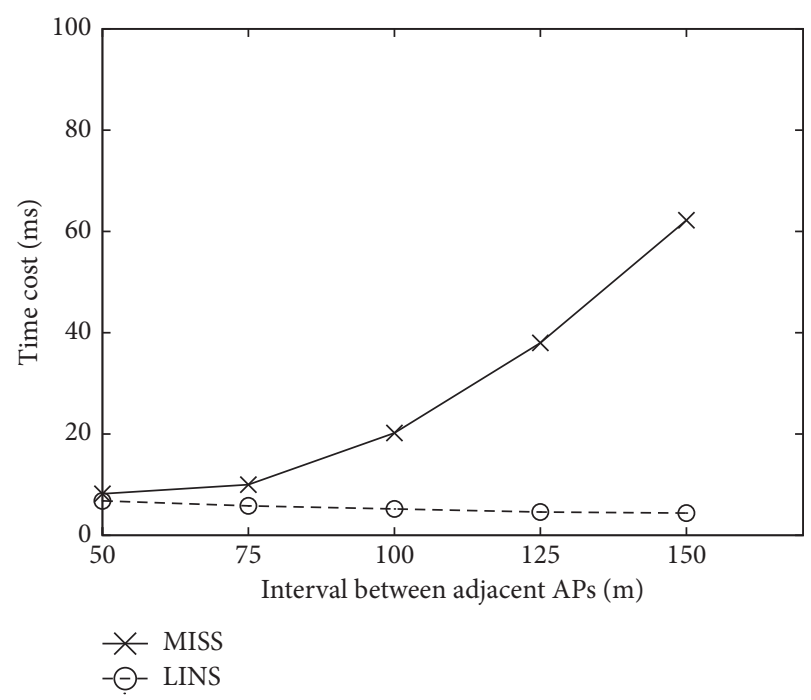

FIGURE 8: The average time cost of MISS and LINS when the distance between the adjacent APs varies.

scheduling algorithms have similar performance in OTS, LINS is more suitable for practical use. Hence, in the following simulations, only LINS is compared with the benchmark SUM.

The residual bandwidth is provided to secondary users, which should be maximized to improve network utilization. When the number of the primary users changes, the residual bandwidths of LINS and SUM are demonstrated in Figure 10. Thanks to the concurrent transmission scheduled by LINS, the residual bandwidth of LINS decreases much slower than that of SUM.

In Figure 11, the residual bandwidth of LINS increases slightly when the APs are deployed sparsely, while the AP density does not affect the residual bandwidth of SUM.

As shown in Figure 12, when the demanded bandwidths of the primary users increase, the residual bandwidth of 


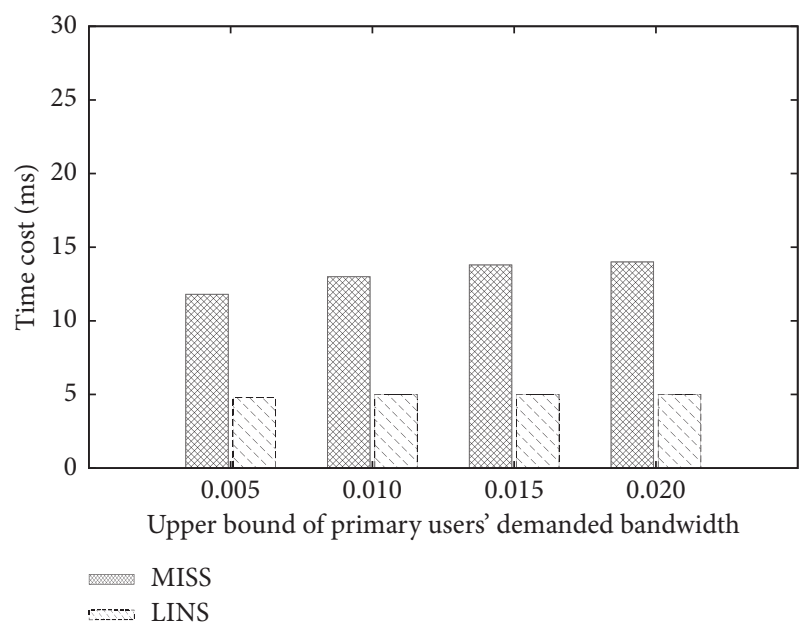

FIgUre 9: The average time cost of MISS and LINS when the demanded bandwidth of primary users varies.

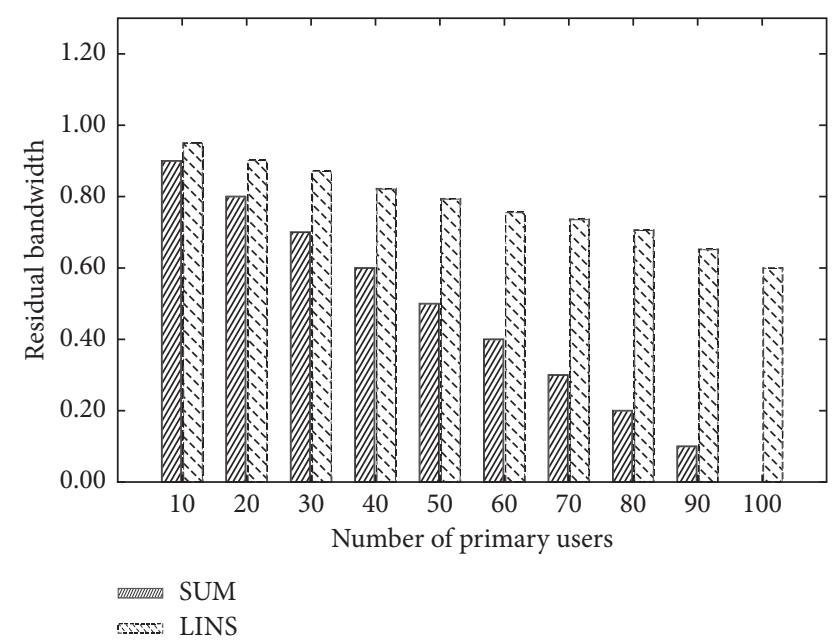

FIGURE 10: The residual bandwidth of LINS and SUM when the number of primary users varies.

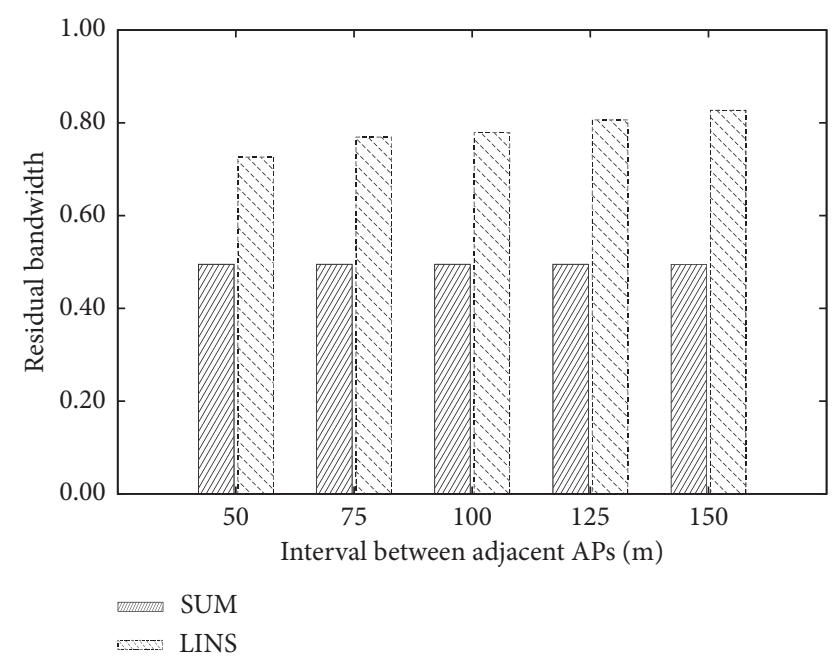

FIGURE 11: The residual bandwidth of LINS and SUM when the distance between the adjacent APs varies.

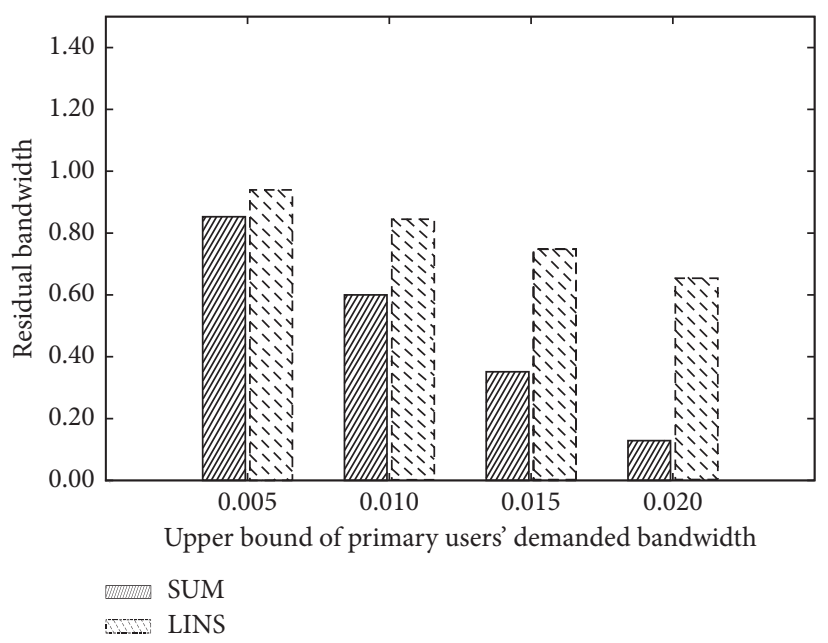

FIgURE 12: The residual bandwidth of LINS and SUM when the demanded bandwidth of primary users varies.

SUM decreases greatly. However, LINS has a slower rate of descent, owing to its scheduling.

From Figures 10-12, a conclusion can be drawn that the network utilization is improved due to transmission scheduling in WiFi networks.

\section{Conclusion}

To provide differentiated and guaranteed downlink bandwidth for devices in smart spaces, a framework of wireless virtual access network was designed in software-defined WiFi networks. Based on this framework, two scheduling algorithms, that is, MISS and LINS, were proposed to maximize the network utilization. The MISS algorithm employed the maximum independent set in the conflicted graph to increase concurrent transmissions of AP groups. The LINS algorithm scheduled the AP groups in a linear order, which had lower computation complexity than MISS. Extensive simulation experiments were conducted, and the results indicated that both algorithms scheduled wireless virtual access networks effectively. Nevertheless, the LINS algorithm was more suitable for practical use due to its high efficiency.

\section{Data Availability}

The data used to support the findings of the study are available from the first author upon request (lvpin@ gxu.edu.cn).

\section{Conflicts of Interest}

The authors declare that there are no conflicts of interest regarding the publication of this paper.

\section{Acknowledgments}

This research was funded by the Special Funds for Guangxi BaGui Scholars, National Natural Science Foundation of China (61402513 and 62062008), Guangxi Natural Science 
Foundation (2019JJA170045, 2018JJA170194, 2018JJA170028, and 2016JJB170040), and Scientific Research Foundation of Guangxi University (XGZ150322).

\section{References}

[1] J. Sheth and B. Dezfouli, "Enhancing the energy-efficiency and timeliness of IoT communication in WiFi networks," IEEE Internet of Things Journal, vol. 6, no. 5, pp. 9085-9097, 2019.

[2] Y. Sun, J. Chen, Y. Tang, and C. Yanjia, "Energy modeling of IoT mobile terminals on WiFi environmental impacts $\dagger$," Sensors, vol. 18, no. 6, p. 1728, 2018.

[3] J. Yang, H. Zou, H. Jiang, and L. Xie, "Device-free occupant activity sensing using WiFi-enabled IoT devices for smart homes," IEEE Internet of Things Journal, vol. 5, no. 5, pp. 3991-4002, 2018.

[4] H. Zou, Y. Zhou, J. Yang, and C. J. Spanos, "Towards occupant activity driven smart buildings via WiFi-enabled IoT devices and deep learning," Energy and Buildings, vol. 177, pp. 12-22, 2018.

[5] C. Del-Valle-Soto, L. J. Valdivia, R. Velázquez, L. RizoDominguez, and J.-C. López-Pimentel, "Smart campus: an experimental performance comparison of collaborative and cooperative schemes for wireless sensor network," Energies, vol. 12, no. 16, p. 3135, 2019.

[6] C. Liang and F. R. Yu, "Wireless network virtualization: a survey, some research issues and challenges," IEEE Communications Surveys \& Tutorials, vol. 17, no. 1, pp. 358-380, 2015.

[7] T. M. Ho, N. H. Tran, S. M. A. Kazmi, Z. Han, and C. S. Hong, "Wireless network virtualization with non-orthogonal multiple access," in Proceedings of the NOMS 2018-2018 IEEE/ IFIP Network Operations and Management Symposium (NOMS), Taipei, Taiwan, April 2018.

[8] P. Lv, X. Wang, and M. Xu, "Virtual access network embedding in wireless mesh networks," Ad Hoc Networks, vol. 10, no. 7, pp. 1362-1378, 2012.

[9] Y. Amir, C. Danilov, R. Musualoiu-Elefteri, and N. Rivera, "The SMesh wireless mesh network," ACM Transactions on Computer Systems (TOCS), vol. 28, no. 3, p. 6, 2010.

[10] H. Wen, P. K. Tiwary, and T. Le-Ngoc, Wireless Virtualization, Springer, Berlin, Germany, 2013.

[11] M. Yang, Y. Li, D. Jin, L. Zeng, X. Wu, and A. V. Vasilakos, "Software-defined and virtualized future mobile and wireless networks: a survey," Mobile Networks and Applications, vol. 20, no. 1, pp. 4-18, 2015.

[12] N. Zhang, P. Yang, S. Zhang et al., "Software defined networking enabled wireless network virtualization: challenges and solutions," IEEE Network, vol. 31, no. 5, pp. 42-49, 2017.

[13] D. B. Rawat, "Fusion of software defined networking, edge computing, and blockchain technology for wireless network virtualization," IEEE Communications Magazine, vol. 57, no. 10, pp. 50-55, 2019.

[14] K. M. Park and C. K. Kim, "A framework for virtual network embedding in wireless networks," in Proceedings of the 4th International Conference on Future Internet Technologies, Seoul, Republic of Korea, June 2009.

[15] P. Lv, X. Wang, X. Xue, and M. Xu, "SWIMMING: seamless and efficient WiFi-based internet access from moving vehicles," IEEE Transactions on Mobile Computing, vol. 14, no. 5, pp. 1085-1097, 2015.

[16] P. Lv, X. Wang, Y. Yang, and M. Xu, "Network virtualization for smart grid communications," IEEE Systems Journal, vol. 8, no. 2, pp. 471-482, 2013.
[17] A. Banchs, P. Serrano, P. Patras, and M. Natkaniec, "Providing throughput and fairness guarantees in virtualized WLANs through control theory," Mobile Networks and Applications, vol. 17, no. 4, pp. 435-446, 2012.

[18] A. Checco and D. J. Leith, "Fair virtualization of 802.11 networks," IEEE/ACM Transactions on Networking, vol. 23, no. 1, pp. 148-160, 2015.

[19] Y. Zhao, D. Guo, J. Xu et al., "CATS: Cooperative allocation of tasks and scheduling of sampling intervals for maximizing data sharing in WSNs," ACM Transactions on Sensor Networks (TOSN), vol. 12, no. 4, pp. 1-26, 2016. 